\title{
Spatial Domain Method for Fusing Multi-Focus Images using Measure of Fuzziness
}

\author{
R. Maruthi \\ Assistant Professor, MCA Dept \\ SoMCA, SSN College of Engineering \\ OMR, Kalavakkam, Chennai-603 110
}

\begin{abstract}
A simple and effective image fusion technique has been designed to convey all the meaningful information from the images of the same scene with diverse focuses into a final composite image. This paper presents an alternate approach to the problem of multi-focus image fusion using index of fuzziness. The fusion performance of the proposed method has been evaluated using an objective measure. Experimental results on several pairs of images indicate that the proposed method works well when compared to some of the image fusion methods.
\end{abstract}

\section{Keywords}

Multi-focus images, index of fuzziness, image fusion, spatial domain.

\section{INTRODUCTION}

Image fusion is an important research area for the militarily significant states world wide. It is used in recognizing a ground/air force vehicle, remote sensing, medical imaging, optical microscopy etc., [1]. The purpose of image fusion is to ultimately obtain the accurate as well as abundant information from the fused images, which is not available in a single image. Multi-focus image fusion is a process of fusing images that are taken under different focal settings in the same viewpoint. The images which are obtained in diverse focuses are fused to produce an image, which is focused in all areas with adequate information.

Various approaches have been found in the literature for fusing multi-focus images. Simple techniques, in which the fusion operation is performed directly on the source images like weighted average method, often have serious side effects like reduction in the contrast of the fused image. Most of the image fusion techniques were based on wavelets [1,2] principle component analysis (PCA)[1] and various adaptive schemes[3, $4,8,11]$. It involves huge computations using floating point arithmetic and thus requires a lot of time and memory space [14].

According to the combination mechanisms, image fusion methods can be generally divided into three categories namely, pixel level, feature level, and decision level. The feature level and decision level fusion may lead to a loss of information during the fusion process [9]. The approaches for image fusion can be classified as spatial domain methods and frequency domain methods [12]. Most of the multi-focus image fusion methods are based on frequency domain approaches and very few algorithms have been suggested in spatial domain.
Therefore, a pixel level fusion in spatial domain for fusing multi-focus images is focused in this paper.

An improved method in spatial domain is to compute the degree of focus for each pixel using various focus measures in multifocus images. A critical issue in the design of multi-focus image fusion algorithms is to define activity measures or focus measures that can evaluate and compare the local information content of multiple images [12]. The conventional focus measures found in the earlier works include, spatial frequency $[1,2,3,5,6,14,17]$, pixel visibility $[3,4,5,17]$, uniform parameter [6], variance[17], energy of image gradient[17], energy of laplacian of image [17] etc,. In this paper, a new alternate approach to the problem of multi-focus image fusion based on index of fuzziness as a focus measure has been suggested. This method is computationally simple and can be applied in real time applications. Experimental results show that it outperforms the spatial frequency based method and pixel level maximum and minimum method.

The rest of the paper is organized as follows. An introduction to the index of fuzziness is given in section 2. The proposed fusion scheme is described in section 3. Experimental results are given in the section 4 followed by concluding remark.

\section{INDEX OF FUZZINESS}

The increase or decrease in image fuzziness can be used in image processing tasks such as enhancement, segmentation and classification [15]. The index of fuzziness (IF) measures the information level in an image. For an $\mathrm{M}^{*} \mathrm{~N}$ image block, the index of fuzziness is defined as follows.

$$
I F=\frac{2}{M N} \sum_{m} \sum_{n} \min \left(\mu_{m n}, 1-\mu_{m n}\right)
$$

Where the size of an image is $\mathrm{MxN}$ and $\mu$ is the mean value of the image block. The index of fuzziness is calculated regarding the difference between the membership values $(\mu)$ and their complements [15]. The membership values can be chosen based on the application.

The experiments have been performed on four sets of various versions of blurred images. Fig-1(a) is the original image and 1(b) to 1 (d) shows the degraded versions of the images after blurring with the Gaussian of radius $0.5,1.0,1.5$ and 2.0 respectively. It is clear from the table- 1 and table-2, the index of fuzziness (IF) decreases with increase in blurriness. The same experiment is repeated with different set of images. This reveals that the index of fuzziness is used to measure the depth of field 
in an image and is used in estimating the quality of the blurred or defocused images.
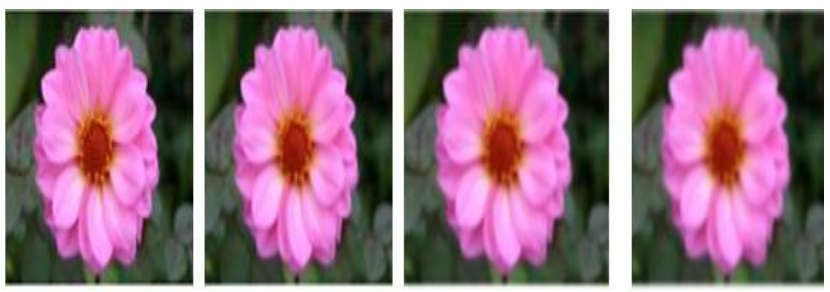

Fig-1(a)Flower

(b) Blur,0.5 r (c)Blur,1.0 r

d) Blur $1.5 \mathrm{r}$

Table-1 Index of Fuzziness value of the image blocks in Fig-1

\begin{tabular}{|c|c|c|c|c|}
\hline Measure & Fig-1 (a) & Fig-1 (b) & Fig-1 (c) & Fig-1 (d ) \\
\hline IF(Flower) & 22.92 & 22.90 & 22.87 & 22.84 \\
\hline
\end{tabular}

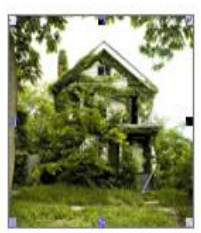

Fig-2(a)Tree

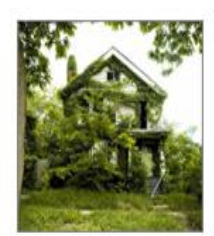

(b) Blur,0.5 r (c)Blur,1.0 r

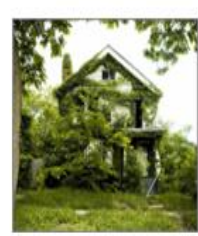

d) Blur $1.5 \mathrm{r}$
Table-2 Index of Fuzziness value of the image blocks in Fig-2

\begin{tabular}{|c|c|c|c|c|}
\hline Measure & Fig-2 (a) & Fig-2 (b) & Fig-2 (c) & Fig-2 (d ) \\
\hline IF(Tree) & 21.54 & 21.51 & 21.48 & 21.46 \\
\hline
\end{tabular}

From the Table- 1 and Table-2, it is found that the value of the index of fuzziness (IF) decreases with increase in blurring. The IF value is calculated on the various set of images and it is found to be the same. Hence the index of fuzziness is found to be an image quality metric for blurred images. This reveals that, the index of fuzziness is used to measure the depth information in defocused images.

\section{MULTI-FOCUS IMAGE FUSION}

The diagram for the fusion scheme is given in Fig-3. The fusion of multi-focus (left and right focus) registered (same co-ordinate system) images of the same size is considered for the fusion process. The fuzzification is done on the source images of size $\mathrm{M}^{*} \mathrm{~N}$ by calculating the index of fuzziness(IF) for each block of source images regarding the membership values and their complements. An appropriate membership value is chosen based on the application. The mean value of each sub window of the source images are taken as membership values. The index of fuzziness (IF) is calculated for each block of the source images and the rule evaluation is done by comparing the index of fuzziness (IF) of the first image with the second image. Based on the values of the index of fuzziness the resultant image is formed. The de-fuzzification is performed based on the resultant image to obtain crisp results. This step is performed here by again comparing the fused image pixel values with the source images to get the enhanced fused image.

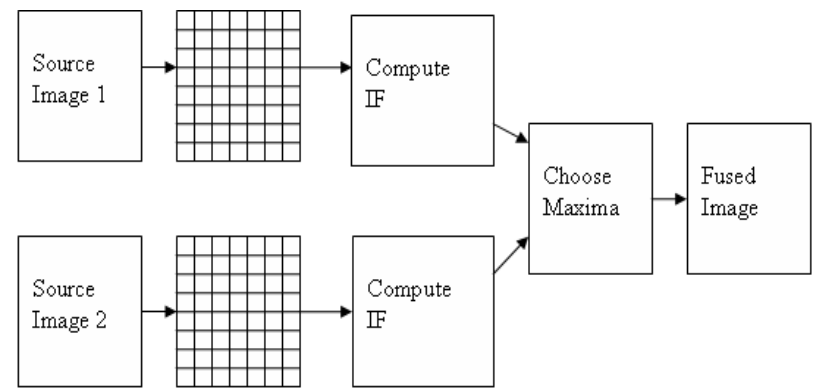

Fig-3 Diagrammatic representation of the fusion process using measure of fuzziness

The algorithm works as follows.

1. Decompose the source images A and B into sub-blocks of size $\mathrm{M}^{*} \mathrm{~N}$ and denote the ith block as $\mathrm{Ai}$ and $\mathrm{Bi}$ respectively. The block size can be $3 * 3$ or $4 * 4$ etc. But the quality of the fused image will decrease with increase in block size.

2. Compute the membership values and their complements by choosing an appropriate membership function. Here the membership function used is the mean value of each subblock.

3. Compute the IF using the equation-(1) for each sub-block using the membership values and their complements and denote the IF's of $\mathrm{Ai}$ and $\mathrm{Bi}$ as IFAi and IFBi respectively.

4. Compare the IF's of the two corresponding sub-blocks Ai and $\mathrm{Bi}$ and construct the ith block $\mathrm{F}$ of the fused image as follows

$$
\begin{aligned}
& \text { If IFAi > IFBi } \\
& \mathrm{Fi}=\mathrm{Ai} \\
& \text { If IFAi }<\text { IFBi } \\
& \mathrm{Fi}=\mathrm{Bi} \\
& \text { If IFAi = IFBi } \\
& \mathrm{Fi}=\mathrm{Ai} \text { or } \mathrm{Bi}
\end{aligned}
$$

\section{EXPERIMENTAL RESULTS}

Experiments have been performed in four set of images. The source left and right focus images were given in the Figures- 4, 5, 6, 7 (a) and (b). The resultant fused images obtained using the proposed method (IF) and the compared methods (SF and MM) were given in the figures- $4,5,6,7$ (c) to (e). The brief description of the compared methods is given below. The spatial frequency which measures the overall activity level in an image $[3,5,6,14,16,17]$ is used in the fusion process as well as in the evaluation of fusion performance with no ground truth. For an $\mathrm{M} * \mathrm{~N}$ block of an image, it is calculated as follows,

$$
\mathrm{SF}=\sqrt{(R F)^{2}+(C F)^{2}}
$$

Where RF and CF are the row frequency and column frequency respectively. 


$$
\begin{gathered}
\mathrm{RF}=\sqrt{\frac{1}{M N} \sum_{m=1}^{m} \sum_{n=2}^{n}[F(m, n)-F(m, n-1)]^{2}} \\
\mathrm{CF}=\sqrt{\frac{1}{M N} \sum_{n=1}^{n} \sum_{m=2}^{m}[F(m, n)-F(m-1, n)]^{2}}
\end{gathered}
$$

Higher the value of spatial frequency, higher will be the image quality.

The simplest method of image fusion is pixel level maximum or minimum. In this method pixel level fusion is done in which the intensity values of two source images is compared pixel by pixel and the one with the higher value is taken in the fused image [5]. Table-1 gives the spatial frequency (SF) values of the fused images.

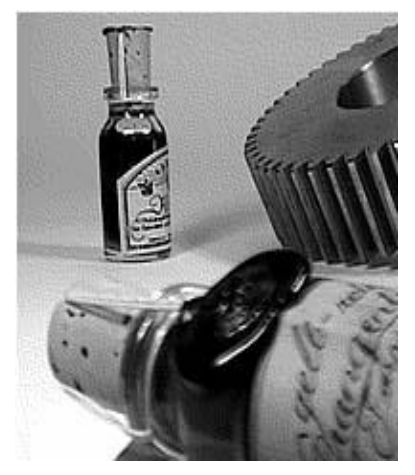

Fig-4 (a)Bottle- Left focus image

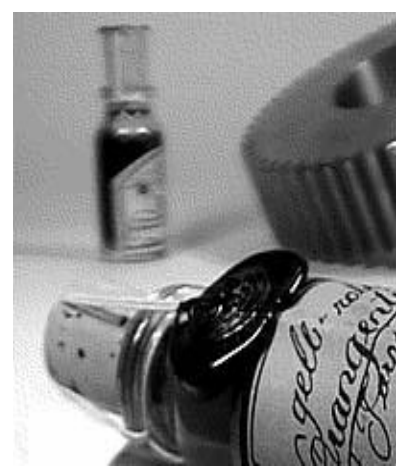

b) Right focus image
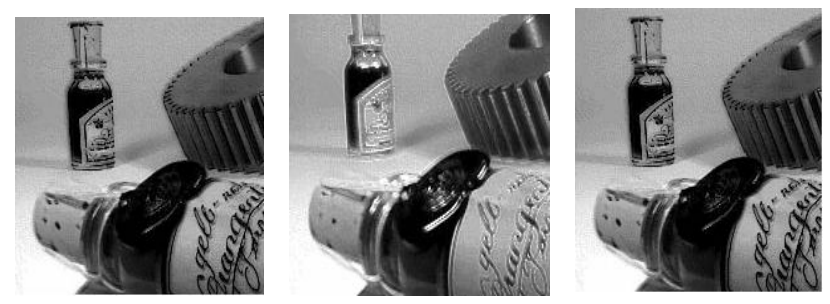

(c)Fused Image-IF (d)Fused Image-SF e)Fused-Image-MM
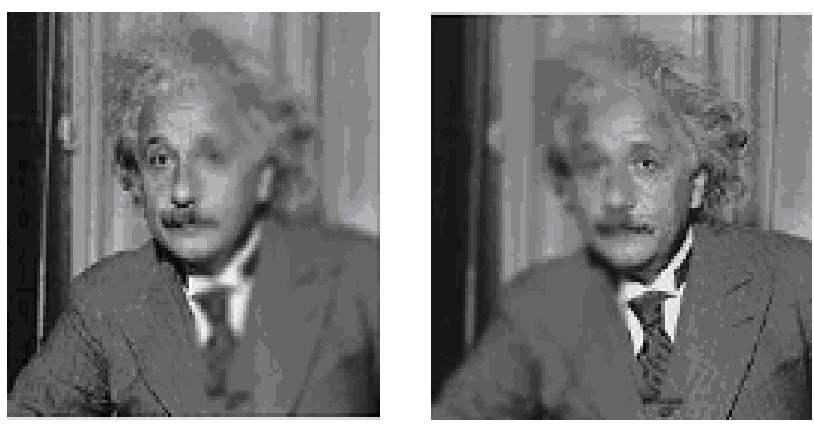

Fig-5 (a) Oldman-Right Focus image (b) Left focus image
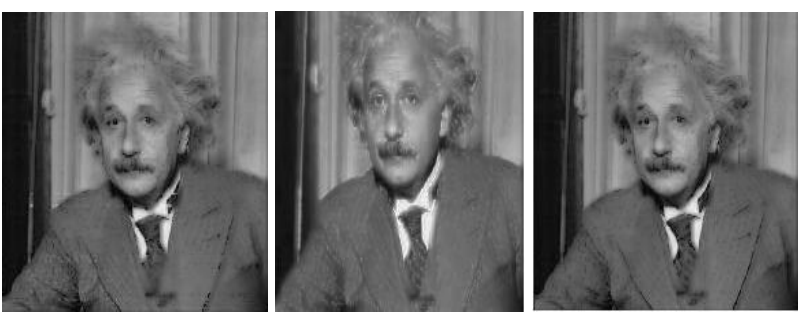

(c)Fused Image-IF (d) Fused Image-SF e)Fused-ImageMM
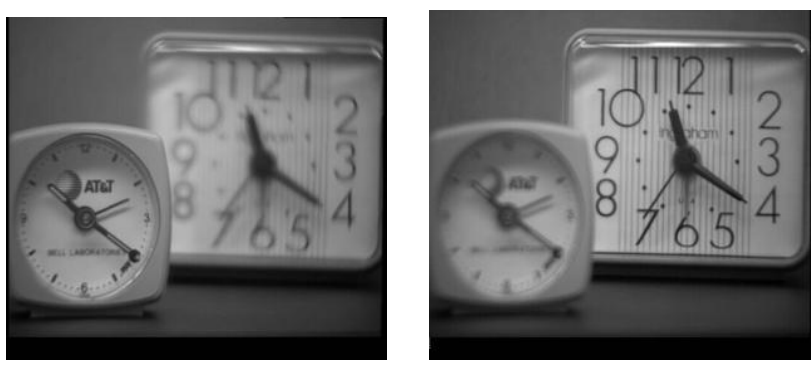

Fig-6-Clock (a) Right Focus image

(b) Left focus image
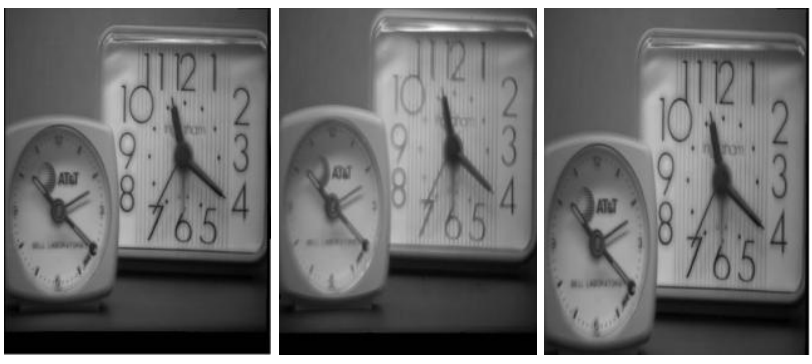

(c)Fused image-IF (d) Fused image -SF e) Fused-ImageMM
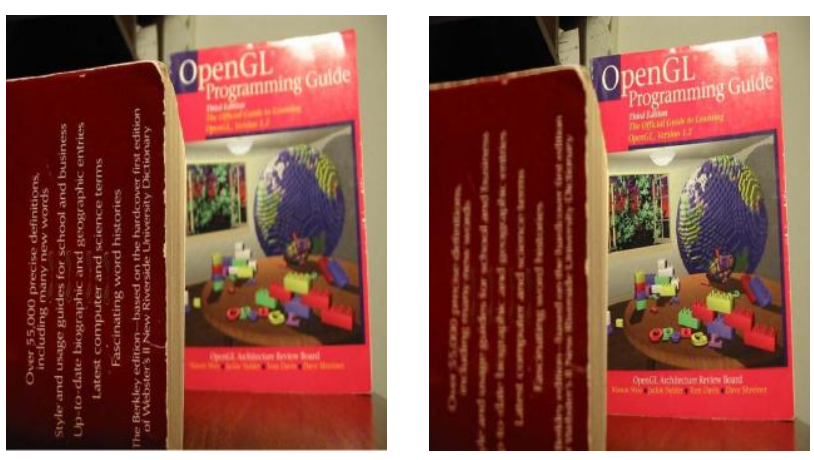

Fig-7 (a)Book-Right Focus image

(b) Left focus image
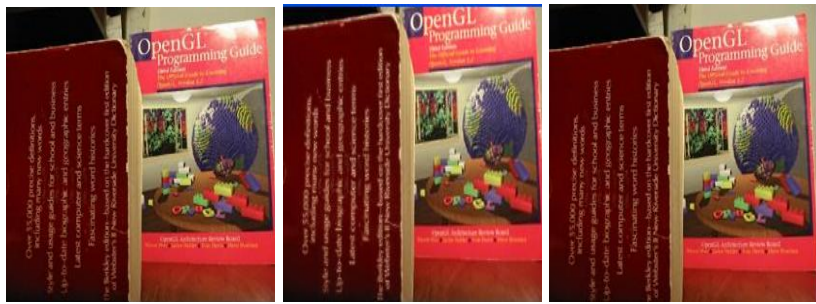

(c) Fused Image-IF (d) Fused Image -SF e) Fused-ImageMM 
Table-3 Spatial frequencies value for the fused images

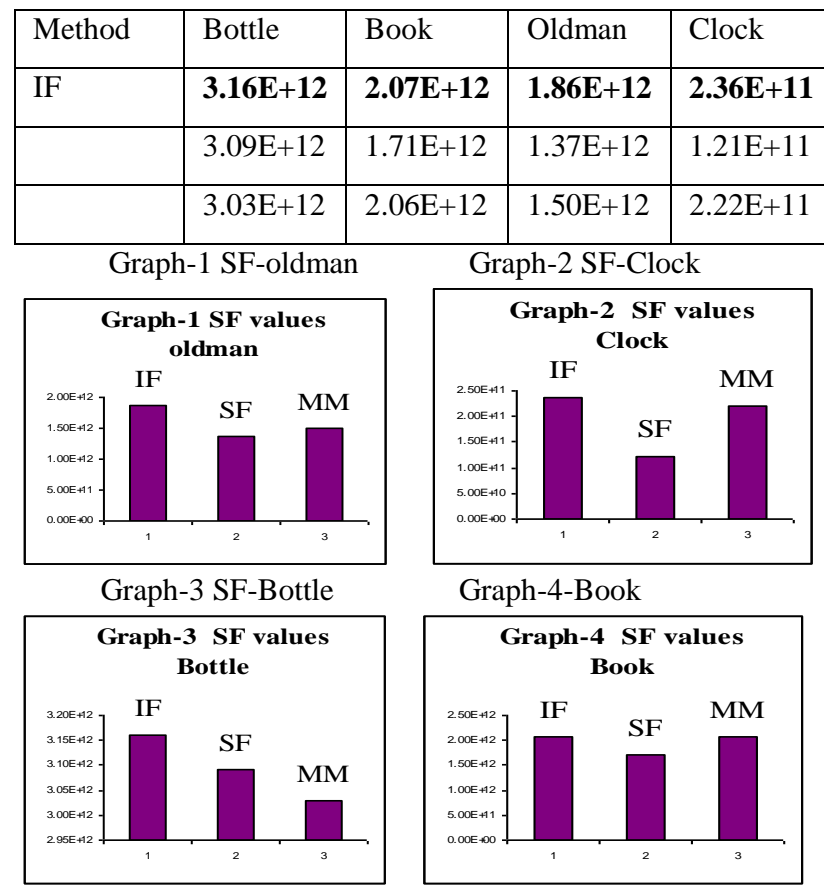

From the Figures-4,5,6 \& 7 (c) to (e) and graphs-1,2,3 and 4, it is clear that the fused images with the proposed method (IF) is visually and quantitatively effective than the spatial frequency(SF) and pixel level maximum and minimum methods(MM).

\section{CONCLUSION}

In this paper, a new alternate approach to the problem of fusing multi-focus images has been proposed. It is computationally simple and can be applied in real time applications. Comparison with the spatial frequency method and pixel level maximum and minimum method shows that the proposed method outperforms the other two methods. The proposed approach can be applied to other categories of image fusion using various measures of fuzziness and image information. A new combinational approach can be developed based on several other focus measures

\section{REFERENCES}

[1]. Dheeraj Agrawal, Dr.Al-Dahoud Ali, Dr.J.Singhai (2009). A Modified Partition Fusion Technique of Multifocus Images For Improved Image Quality, UbiCC Journal - Volume 4 No. 3Special Issue on ICIT 2009 Conference Bioinformatics and Image.

[2]. Richang Hong, Chao Wang, Meng Wang and Fuming Sun(2009). Salience Preserving Multi Focus Image Fusion With Dynamic Range Compression, International Journal of Innovative Comp.Info. and Control ICIC International c 02009 ISSN 1349-4198 Volume 5, Number 8, August 2009.

[3]. Shutao Li, James T.Kwok, Yaonan Wang (2002), Multifocus image fusion using artificial neural networks, Pattern Recognition Letters 23 (2002) 985-997.

[4]. Zhenhua Li; Zhongliang Jing; Gang Liu; Shaoyuan Sun; Henry Leung (2003). Pixel visibility based multifocus image fusion, Neural Networks and Signal Processing, Proceedings of the International Conference on Volume 2, Issue, 14-17 Dec.2003 Page(s): 1050 - 1053 Vol.2

[5]. M.E.Ravinandan and Jharna Majumdar (2005). Feature based Image fusion Proceedings of the International Conference on Cognition and Recognition, 2005.

[6]. Xinman, Zhang, Juqianghan, Peifeiiu (2005). Restoration and fusion optimization scheme for multi-focus image using genetic search strategies, Optica Aplicata Vol 1 No.4, 2005

[7]. Martina Dankova and Radek Valasek (2006). Fully fuzzy transform and the problem of image fusion., Journal of Electrical Engineering, Vol. 57, Issue. 12.

[8]. Ishita De, Bhabatosh Chanda and Buddhajyoti Chattopadhyay (2006). Enhancing effective depth-of-field by image fusion using mathematical morphology, Image and Vision Computing Volume 24, Issue 12, 1 December 2006, Pages 1278-1287.

[7]. Zheng, S.; Liu, J.; Zhu, G.; Tian, J(2006). A support value transform-based multi focus image fusion method, Signal Processing, 2006 8th International Conference on Volume 2, 2006.

[8]. Lijian Zhou, Guangrong Ji, Chan gjiang Shi, Chen Feng and Rui Nian (2006). A Multi-focus Image Fusion Method Based on Image Information Features and the Artificial Neural Networks, Lecture Notes in Control and Information Sciences, Pp 747-752, Volume 344/2006.

[9]. Wei Huang, Zhong Liang, Jing (2007). Multifocus image fusion using Pulse coupled Neural Network, Vol 28, Issue 9 PP 1123-1132, Pattern Recognition Letters, 2007.

[10]. J. Yang, Y. Ma, W. Yao, W. T. Lu (2008). A Spatial domain and frequency domain integrated approach to fusion multifocus images, ISPRS Congress Beijing 2008 Proceedings of Commission VII.

[11].Shutao Li, Bin Yang(2008).Multi-focus image fusion using region segmentation and spatial frequency, Image and Vision Computing Volume 26 , Issue 7 (July 2008) Pages 971-979 ISSN:0262-8856.

[12]. Jun Kong, Kaiyuan Zheng, Jingbo Zhang, Xue Feng(2008). Multi-focusImage Fusion Using Spatial Frequency and Genetic Algorithm, International Journal of Computer Science and Network Security, Vol.8 No.2, February 2008.

[13] V.P.S.Naidu, J.R Raol (2008). Pixel level Image Fusion using wavelets and PCA Defense Science Journal Vol 58 No.3, Pp 338-352, May 2008.

[14]. Wei Wang, Peng -Lang Shui and Xiang-chu Feng(2008). Variational Models for Fusion and Denoising of Multifocus Images, IEEE Signal Processing LettersVol 152008.

[15]. Hamid R. Tizhoosh (1997), Introduction in Theory and Practice, Fuzzy Imag Processing, Springer Verlag.

[16]. Shutao Li, James T. Kwok and Yaonan Wang (2001). Combination of images with diverse focuses using the spatial frequency, Information Fusion ,Volume 2, Issue 3, Pages 169-176.

[17]. Yingjie Zhang and Liing Ge(2009). An efficient fusion scheme for multi-focus images by using blurring measure, Digital signal Processing, volume 19, Issue 2,March 2009,Pages 186-19. 